\title{
Efecto de las TIC sobre la gestión de las empresas hoteleras afiliadas a Cotelco de Bucaramanga - Santander, Colombia-
}

Diana Oliveros Contreras ${ }^{1}$ Universidad Autónoma de Bucaramanga doliveros364@unab.edu.co

Gabriel Mauricio Martínez ${ }^{2}$ Universidad Autónoma de Bucaramanga gmartinez714@unab.edu.co

DOI: https://doi.org/10.21158/01208160.n83.2017.1827

Fecha de recepción: 19 de enero de 2017

Fecha de aprobación: 12 de junio de 2017

Cómo citar este artículo / To reference this article / Comment citer cet article / Para citar este artigo:

Oliveros, D. \& Martínez, G. (2017). Efecto de las TIC sobre la gestión de las empresas hoteleras afiliadas a Cotelco de Bucaramanga -Santander, Colombia-. Revista EAN, 83, pp 15-30. https://doi.org/10.21158/01208160.n83.2017.1827

\section{Resumen}

El crecimiento del sector hotelero en Colombia es un fenómeno que se ha desarrollado recientemente como consecuencia de la globalización, que ha conllevado cambios en la oferta, la demanda, las preferencias de los clientes, así como en la rapidez en el desarrollo y la propagación de las tecnologías de la información y de la comunicación (TIC), y en el caso de los hoteles, como un instrumento para responder ágilmente a los requerimientos del mercado y en actividades de soporte. Esta investigación busca evaluar el efecto de la incorporación de las TIC sobre la gestión de los hoteles afiliados a Cotelco en Bucaramanga -Santander-. Se encontró que el uso de las TIC facilita la gestión y mejora la competitividad, dado que les permite a los hoteles responder de una manera más efectiva a sus clientes y ofrecer servicios de forma más rápida, lo cual conlleva una mejora en la calidad del servicio prestado y en la satisfacción del cliente.

\section{Palabras clave}

TIC, Cotelco, eficiencia, hoteles, gestión.

\footnotetext{
${ }^{1}$ Ingeniera industrial por la Universidad de Pamplona, magíster en Gestión de la Calidad para las Organizaciones por Bureau Veritas y doctora en Sistemas Flexibles de Dirección de Empresas por la Universidad Pública de Navarra. Docente de la Universidad Autónoma de Bucaramanga.

${ }^{2}$ Ingeniero industrial por la Fundación Universidad de América, magíster en Logistics and Supply Chain Management por la Lancaster University y doctor en ingeniería por la Universidad Autónoma de Bucaramanga. Docente de la Universidad Autónoma de Bucaramanga. ORCID: http://orcid.org/0000-0002-2859-5371
} 


\title{
Impact of ICT on the management of hotel companies affiliated to Cotelco in Bucaramanga, -Santander, Colombia-
}

\begin{abstract}
The rising growth of the tourist hotel sector in Colombia is a phenomenon that has developed recently as a consequence of Globalization. It has allowed changes in offer, demand, customers 'preferences, as well as fast development and spread of information and communication technologies, specifically in hotels as a tool to respond fast to marketing requirements and other supporting activities. This study aims at evaluating the impact of the use of ICT on the management of hotels affiliated to Cotelco in Bucaramanga, Santander. This study also showed that the application of ICT facilitates management and improvement of competitiveness due to the fact that they allow hotels to respond to customers effectively and to offer fast services, which lead to improving quality service and satisfying customers' needs.
\end{abstract}

Key words. ICT, Cotelco, efficiency, hotels, management.

\section{Effets des TIC sur la gestion des entreprises hôtelières adhérentes à Cotelco Bucaramanga - Santander, Colombie-}

Résumé. La croissance du secteur hôtelier colombien est un phénomène qui s'est accéléré récemment du fait de la mondialisation et qui connait des changements en termes d'offre, de demande, de préférences clients, de rapidité de développement et de propagation des technologies de l'information et de la communication (TIC) qui sont un instrument de réponse efficient aux requêtes du marché et aux activités de support. Cette étude tente d'évaluer l'effet de l'incorporation des TIC à la gestion des hôtels adhérents à Cotelco Bucaramanga-Santander-. Les résultats semblent montrer que l'usage des TIC facilite la gestion et renforce la compétitivité des hôtels en consolidant les taux de réponse-client et les offres de services tout en entrainant une satisfaction du client en hausse.

Mots clefs. TIC, Cotelco, efficacité, hôtel, gestion.

\section{Efeito das TIC na gestão das empresas hoteleiras filiadas a Cotelco de Bucaramanga -Santander, Colômbia-}

Resumo. O crescimento do setor hoteleiro na Colômbia é um fenômeno recentemente desenvolvido como resultado da globalização, o que tem levado a mudanças na oferta, na demanda, nas preferências dos clientes, assim como ao rápido desenvolvimento e propagação das tecnologias da informação e comunicação (TIC) que, no caso dos hotéis, se tornou um instrumento para responder rapidamente às exigências do mercado como atividades de suporte. Esta pesquisa procura avaliar o efeito da incorporação das TIC na gestão dos hotéis filiados a Cotelco em Bucaramanga -Santander-. Verificou-se que o uso das TIC facilita o gerenciamento e melhora a competitividade, uma vez que permite aos hotéis responder de forma mais eficaz aos seus clientes e oferecer serviços mais rapidamente, o que leva a uma melhoria na qualidade do serviço prestado e na satisfação do cliente.

Palavras-chave. TIC, Cotelco, eficiência, hotéis, gestão. 


\section{Introducción}

$\mathbf{L}^{2}$ a acelerada evolución tecnológica y la globalización han hecho que las tecnologías de la información y de la comunicación (TIC) faciliten nuestra vida. Están presentes en la mayoría de las actividades que realizamos: educación, comunicación, ocio, en la forma de relacionarnos con los demás y en el mundo de los negocios. Las últimas décadas han sido testigo del papel fundamental que han desempeñado las tecnologías como motor de cambio cultural, político y económico de las sociedades. Según el informe Talent Mobility 2020, The next generation of international assignments tal es la importancia que las tecnologías tienen en la sociedad y en el mercado, que quienes no sepan subirse a la ola tecnológica, no podrán sobrevivir. La globalización de la economía y el conocimiento suponen día a día una oportunidad y a la vez un reto para el desarrollo empresarial, si las pequeñas, medianas y grandes empresas no adoptan este tipo de iniciativas, no podrán mantenerse en el tiempo. Las TIC permiten un fácil y rápido acceso a los recursos contribuyendo a mejorar la gestión, la productividad y la competitividad en los negocios.

El sector hotelero ha sido uno de los sectores en los cuales se han incorporado las TIC con mayor rapidez, por tanto, el objetivo de esta investigación fue evaluar el efecto de la incorporación de las TIC sobre la gestión de los hoteles afiliados a la Asociación Hotelera y Turística de Colombia (Cotelco) en Bucaramanga - Santander, Colombia-.

El artículo se compone de cuatro secciones. Inicialmente, se aborda el contexto desde la perspectiva del uso de las TIC en empresas en general y en empresas del sector turístico y hotelero. En la segunda sección, se ilustra la metodología utilizada para el levantamiento y análisis de datos. La tercera parte corresponde a los resultados y hallazgos de la investigación. Finalmente, el último apartado enuncia las conclusiones y los aprendizajes del estudio.

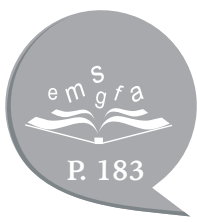

\section{Impacto de las TIC en las empresas}

$\mathbf{L}$ a globalización implica el funcionamiento de los mercados las 24 horas del día y conlleva la necesidad de operar desde cualquier parte del mundo a cualquier hora, suponiendo un cambio en los patrones de consumo. Las TIC se presentan como un instrumento para responder rápidamente a los requerimientos del mercado.
Las tecnologías han representado inversiones económicas bastante importantes no solo en su desarrollo per se, sino en su implementación, uso y adopción en las organizaciones. Estas pueden emplearse a lo largo de toda la cadena de suministro y en cualquier función comercial, no limitándose a un área específica, es decir, desde 
la búsqueda de información en el mercado, el aprovisionamiento, hasta los pagos y el servicio posventa (Leyva, Morales y Heredia, 2015).

Se espera que una más alta inversión en TIC se vea compensada con un mayor rendimiento de las empresas, aunque no todas presentan los mismos resultados necesariamente. Diversos investigadores señalan que invertir recursos en tecnología puede otorgar diversos beneficios a las organizaciones, pero se cuestionan también si, realmente, está inversión mejora la gestión, eleva la eficiencia y la productividad de las organizaciones.

Los primeros aportes científicos sobre el valor estratégico de las tecnologías se divulgan principalmente en la década de 1980. Así, por ejemplo, MacFarlan (1985) y Millar y Porter (1986) manifiestan que las tecnologías permiten mejorar la posición competitiva de las organizaciones que saben obtener beneficio de su uso, añadiendo valor a sus procesos internos y a sus productos. Goldhar y Jelinek (1985) afirman que las empresas que emplean tecnologías pueden obtener ventajas a través de una mayor diferenciación de productos, en otras palabras, de productos personalizados y mejor adaptados al gusto de los clientes. De acuerdo con ellos, esto puede traer consigo mejoras en la producción, las ventas directas, los precios de oportunidad y las ventajas competitivas. Esto último, en particular, al acceder a una mayor variedad de segmentos de mercado.
De la misma forma, Bakos y Treacy (1986) identificaron que las TIC conducen a un incremento de los resultados y a un mejoramiento de la cooperación con clientes y proveedores, así como en la innovación de productos. Morcillo y Bueno (1993) encontraron que las tecnologías favorecen la gestión empresarial mediante la mejora en el funcionamiento, la eficiencia, la productividad, la rentabilidad, la calidad y la innovación que conlleva ventajas competitivas.

Las tecnologías pueden favorecer también la eficiencia interna, debido al extraordinario incremento de la velocidad de comunicación y la fuerte reducción de los costos de comunicación, lo cual permite alcanzar economías de escala y alcance, y facilitar la toma de decisiones; además, favorece un alto grado de eficiencia con el exterior. En el caso de los proveedores, se da un impacto positivo en los costos de búsqueda, negociación y supervisión de un proveedor.

Autores como Kaushik y Singh (2003), Martínez, Majó y Casadesús (2006), Ibarra, González y Cervantes (2016), Sánchez, De Llano y López (2016) y Monroy y Ramos (2016) señalan la importancia de la adopción de las TIC para el mejoramiento de la gestión, la productividad y la competitividad de las empresas. Mithas, Tafti, Bardhan y Goh (2012), por su parte, tras una evaluación de más de cuatrocientas empresas sugieren que las tecnologías tienen un impacto positivo en las ventas y la rentabilidad. Esto, de hecho, en mayor proporción que otras inversiones como la publicidad y la I+D. 


\section{Impacto de las TIC en las empresas turísticas y hoteleras}

$\mathrm{E}$ proceso de tercerización de las econode 1990 y la primera década del siglo XXI en el sector servicios. Las actividades relacionadas con el ocio y el turismo, especialmente, han aumentado de manera creciente su peso en la economía.

El turismo es considerado una fuente importante de generación de ingresos para las naciones, siendo el sector más dinámico dentro de la industria de servicios (Cipolla, F., Nicol, E. y Cipolla-Ficarra, M., 2011). El incremento de lugares como destinos turísticos ha fortificado el interés por el sector y la inversión destinada para su impulso. Este es considerado hoy un importante factor para el desarrollo socioeconómico de los países, y apalanca la creación de empleos y el estímulo a la construcción de nueva infraestructura en búsqueda de la competitividad en un mercado global.

El turismo internacional, en 2014, creció $44 \%, 48$ millones más que en 2013, para alcanzar un nuevo récord total de USD 1.135 millones, registrando el mayor crecimiento desde la crisis económica de 2009. El incremento más alto se registró en América con $8 \%$, seguido de Asia y el Pacífico con $5 \%$, Medio Oriente con $5 \%$, Europa con $3 \%$ y África con $2 \%$ (UNWTO, 2014). En 2013, los ingresos de exportación generados por el turismo internacional alcanzaron un récord de USD1.409 billones, de los cuales los ingresos obtenidos por los destinos de los visitantes internacionales representaron USD1.195 millones.
El turismo internacional representa 30 $\%$ de las exportaciones mundiales de servicios y $6 \%$ de las exportaciones totales, contribución que es similar para los países desarrollados y las economías emergentes. Como categoría de exportación, ocupa el cuarto lugar en el ámbito mundial, después de combustibles, productos químicos y alimentos. De lo anterior, se puede deducir que hoy el turismo se ha configurado como una de las industrias más importantes del mundo por sus efectos en el desarrollo social y económico de regiones o países, y una industria de mayor crecimiento en las últimas décadas (Holjevac, 2003; Jang y Chen, 2008).

Las condiciones de mercado de las empresas turísticas han sido impactadas por la rápida evolución de las TIC, debido a que estas ofrecen herramientas para la gestión y añadir valor a la experiencia del cliente (Buhalis y Law, 2008). Las empresas turísticas han incorporado las TIC tanto en los servicios que prestan a los clientes como en las actividades que les dan soporte, realizando grandes esfuerzos tanto económicos como de tiempo para diseñar, implementar y gestionar aplicaciones, y así ocupar una de las primeras posiciones en materia de adopción y uso de tecnología (eBusiness W@tch, 2006; Observatorio, 2007a, 2007b; Banco de España, 2010), y permitir a las empresas del sector alcanzar una presencia en mercados globales y ofrecer cadenas de valor más flexibles.

Los cambios en el sector turístico están inevitablemente asociados a los desarrollos en nuevas tecnologías e innovaciones estructurales y organizativas (Stamboulis 
y Skayannis, 2003), los cuales han sido radicales en la mayoría de los sectores, aunque de una forma especial en el turístico y en algunos de sus subsectores como el hotelero. La competencia entre los diferentes actores del sector de turismo presiona a los negocios a mantenerse con información actualizada para poder brindar soporte a la gerencia, y lo que es aún más importante, ofrecer asistencia a los posibles clientes con el fin de influenciar su decisión de compra, lo cual se hace posible con el manejo efectivo de las tecnologías (Law, Leung y Au 2009).

Las TIC han generado cambios significativos en el desempeño de las empresas del sector turístico, las cuales, a pesar de su diversidad, en la mayoría de sus procesos, integran actividades intensivas en tecnología e información - p. ej., hoteles, agencias de viajes, empresas de transporte y operadores turísticos-, que afectan no solo a la producción del servicio, sino a su gestión y distribución (Alberca y Parte, 2010).

Gracias a internet es posible ampliar el volumen de clientes, puesto que los usuarios pueden tener acceso a información privilegiada de los principales sitios turísticos, comparar ofertas, leer comentarios de otros usuarios, etc. Los clientes tienen la posibilidad de desarrollar su propio paquete turístico y las empresas pueden individualizar el servicio. Otro aporte importante es la reducción de costos generada por una disminución en el personal administrativo, en los gastos de traslados y en el número de intermediarios y la eliminación de tareas repetitivas. Esto permite alcanzar flexibilidad en los precios

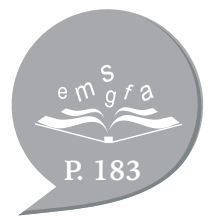
haciéndolos más competitivos con relación a la oferta y la demanda (Caro, 2008; Vilaseca y Torrent, 2004).
Las TIC, en el sector hotelero, han sido reconocidas como una herramienta que genera cambios en la industria (Law, Leung y $\mathrm{Au}, 2013)$. Estas pueden proporcionar multitud de herramientas para mejorar la gestión, facilitar su acceso a nuevos productos y el servicio ofrecido a su clientela. Desde el punto de vista del marketing se pueden conocer mejor las necesidades de los clientes, ofrecer una mejor entrega del servicio, llegar a un mayor número de clientes y optimizar sus recursos logrando aumentar la eficiencia, productividad y competitividad en las organizaciones (Caro, 2008). Los hoteles, con el tiempo, han incorporado tecnologías asociadas a los servicios hoteleros tales como el check-in electrónico, la apertura de habitaciones mediante teléfono móvil o la solicitud de servicios de comida mediante una tableta (González y Gidumal, 2015).

Las aplicaciones más relevantes de las TIC en el sector turístico son utilizadas por las agencias de viajes, aerolíneas, restaurantes, proveedores y hoteles. Estas han sido encaminadas principalmente hacia la implementación de sistemas de reservas en línea, ofertas, promociones y ventas de servicios por internet, así como hacia la interacción y respuesta rápida a las necesidades del cliente, entre otros.

En cuanto a la incidencia de las tecnologías, se encuentran estudios que analizan el efecto de estas en el sector turístico y hotelero. Así, por ejemplo, Botello, Pedraza y Contreras (2015) encontraron para las empresas de servicios que las TIC incrementan la productividad laboral. Estos se apoyan en el correo electrónico y el uso de internet como herramienta para acercarse a los clientes y como plataforma para desarrollar procesos internos de innovación. 
Moliner, Fuentes y Gil (2015) encontraron que el nivel de utilización de las TIC y los beneficios en sus relaciones interorganizacionales son elevados. No obstante, observaron diferencias significativas entre hoteles de diferente categoría. Encontraron que los hoteles de tres estrellas realizan un gran esfuerzo por equipararse con los de categoría superior en su percepción sobre la implantación y los beneficios de las TIC. Así mismo, comentan que cuando los hoteles tienen como cliente principal a agencias minoristas hacen un mayor uso de las TIC, mientras que si se trata de agencias mayoristas lo que destacan son los beneficios percibidos y el grado de desarrollo.

Leyva, Morales y Heredia (2015) realizaron un estudio sobre la importancia de las redes sociales y su impacto en los sistemas de información de los hoteles como una alternativa para aumentar los niveles de productividad y competitividad. El $84 \%$ de los gerentes considera que el uso de la información obtenida de sus redes sociales les ayuda a tomar decisiones que les permiten ser competitivos e innovadores. Las redes sociales se configuran como la herramienta de la web 2.0 más idónea para aquellas estrategias empresariales fundamentadas en la orientación al cliente y la fidelización.

Wichels (2016) estudió cómo las redes sociales han transformado la comunicación hotelera; las redes sociales, como TripAdvisor, Facebook o Twitter están revolucionando los flujos, los procesos y las herramientas de la comunicación turística exigiendo nuevas prácticas en comunicación debido a que las estrategias y herramientas tradicionales se tornan cada vez menos efectivas. Concluye que una adecuada gestión de la comunicación en línea permite a los hoteles conservar una comunicación bidireccional y simétrica con sus clientes, recibir retroalimentación continua y promocionar sus servicios y productos.
Ruiz, Gil y Moliner (2013) señalan la existencia de una relación positiva entre un buen número de aplicaciones tecnológicas en el hotel y la satisfacción del huésped, la intención de recomendar el lugar en internet y de volver a visitar el establecimiento. Rodríguez, Alonso, Rubio y Esteban (2008) indican que la incorporación de las TIC al sector turístico y especialmente internet, han provocado un cambio considerable en los procedimientos de dirección de estas empresas alterando tanto sus relaciones con los agentes externos - proveedores, clientes y competidorescomo su propia operatividad interna.

En el caso del sector turístico en España, Jacob y Bravo (2001) encontraron que los impactos más importantes producidos por la implantación de innovaciones son la mejora de la satisfacción de los clientes, la imagen y la incidencia favorable en la calidad del servicio, mientras que el incremento en la productividad se produce en muy pocas empresas.

En Colombia, Botello, Pedraza y Contreras (2015), quienes analizan los impactos de la implementación de las TIC en el desempeño de las empresas de servicios del país, encontraron que aquellas empresas de servicios que adoptan tecnologías tienen un indicador de ventas por trabajador de $4.3 \%$ por encima del mismo indicador promedio de las empresas analizadas, validando la importancia de este tipo de herramientas para el desempeño de las organizaciones en la actualidad.

Las compañías aéreas, con la distribución de los billetes electrónicos, y las grandes cadenas hoteleras, con la venta de sus servicios, conllevan a pensar que el sector turístico se encuentra a la vanguardia en el uso de tecnologías, sin embargo, es necesario tener en cuenta las empresas pequeñas y medianas 
con recursos limitados que no se pueden permitir grandes inversiones en tecnología.

Buhalis y Law (2008) comentan que los grandes hoteles que pertenecen a cadenas, con muchos departamentos y servicios, tienden a hacer un uso más intensivo de las TIC con relación a los hoteles pequeños que realizan gran parte de sus actividades de forma manual y menos eficiente.

Autores como Lee, Barker y Kandampully (2003), y Sahadev y Islam (2005), encuentran diferencias en las categorías del hotel y la relación en adopción y uso de las tecnologías, siendo los hoteles con mayor categoría los más intensivos.
Sin embargo, el retorno de la inversión en TIC no siempre ha sido el esperado por las empresas. Algunos autores informan que en el sector de la hostelería, en ocasiones, no se aprovecha el potencial que brindan las aplicaciones instaladas (Brown y Stange, 2002; Hensdill, 1998; Martínez, Majó y Casadesús, 2006).

La incorporación de las tecnologías presenta algunas desventajas ante una pérdida de contacto humano y de interacción personal (Jo Bitner, 2001). Los clientes, si bien valoran positivamente la rapidez con que ofrecen las máquinas en la prestación de un servicio, también lo es su deseo de interactuar con otras personas (Meuter, Bitner, Ostrom, y Brown, 2005 y Reinders, Dabholkar y Frambach, 2008).

\section{Metodología}

$\mathrm{P}$ ara medir el efecto de las TIC sobre la gestión y la competitividad de los hoteles, se utilizó una investigación con alcance descriptivo. En primer lugar, nos basamos en los indicadores básicos de tenencia y uso de tecnologías de la información y comunicación del DANE (2012), teniendo en cuenta variables como tenencia en el uso de TIC, actividades realizadas por las empresas que usaron internet y ventas, y compras a través del comercio electrónico.
En segundo lugar, se diseñó una encuesta estructurada que tuvo en cuenta las siguientes variables: número de empleados, nivel educativo, capacitación, nivel de adopción y uso de las TIC, grado de utilización de las TIC —-disposición de correo electrónico, internet, reservas-, tipo de tecnología utilizada - software especializado en clientes, restaurante, recursos humanos, etc.-, seguridad informática y nivel de satisfacción de los clientes con relación a la utilización de las TIC.

La muestra por estudiar fueron 21 hoteles de Bucaramanga y el área metropolitana afiliados a Cotelco -Santander, Colombia-. ${ }^{3}$

\footnotetext{
${ }^{3}$ La información utilizada en la encuesta fue extraída del proyecto titulado Efecto de las TIC sobre la gestión, productividad y competitividad de las empresas hoteleras de Bucaramanga, Santander, financiado por la Universidad Autónoma de Bucaramanga.
} 


\section{Resultados}

\subsection{Indicadores básicos de TIC en empresas en el ámbito nacional}

A continuación, se presenta la información acerca de indicadores básicos de tenencia y uso de TIC en empresas del sector de servicios en Colombia en 2012, para lo cual hay que basarse en la información proporcionada por el DANE.

Según el DANE (2012), existe un uso generalizado de computador e internet en las empresas clasificadas en las actividades complementarias de transporte y comunicaciones, educación superior privada, hoteles y restaurantes, radio, televisión y otros. Las empresas dedicadas a hotelería y restauración reportaron 99.85 \% que suministra computador de escritorio a su personal, $71.34 \%$ portátiles, $35.22 \%$ smartphones y $14.18 \%$ tabletas.
En el desarrollo de las actividades de educación superior privada, registraron la mayor tasa de suministro de computadores portátiles y tabletas con $85.99 \%$ y $28.66 \%$, respectivamente. Las empresas dedicadas a las actividades complementarias de transporte y comunicaciones y radio, televisión y otros, registraron la mayor tasa de suministro de smartphones con $48.97 \%$ y $39.46 \%$. En general, es muy bajo el uso de agendas electrónicas.

Las mayores tasas de empresas con página web se obtienen en los subsectores de educación superior privada con $99.4 \%$ seguido de actividades complementarias de transporte y comunicaciones con un $83.5 \%$, mientras que el subsector de hoteles y restaurantes alcanzó una tasa de $77.6 \%$. El subsector con menor porcentaje de empresas con página web fue el de inmobiliarias, empresariales y de alquiler con $69.2 \%$ (Figura 1).

Figura 1. Tenencia en el uso de TIC.

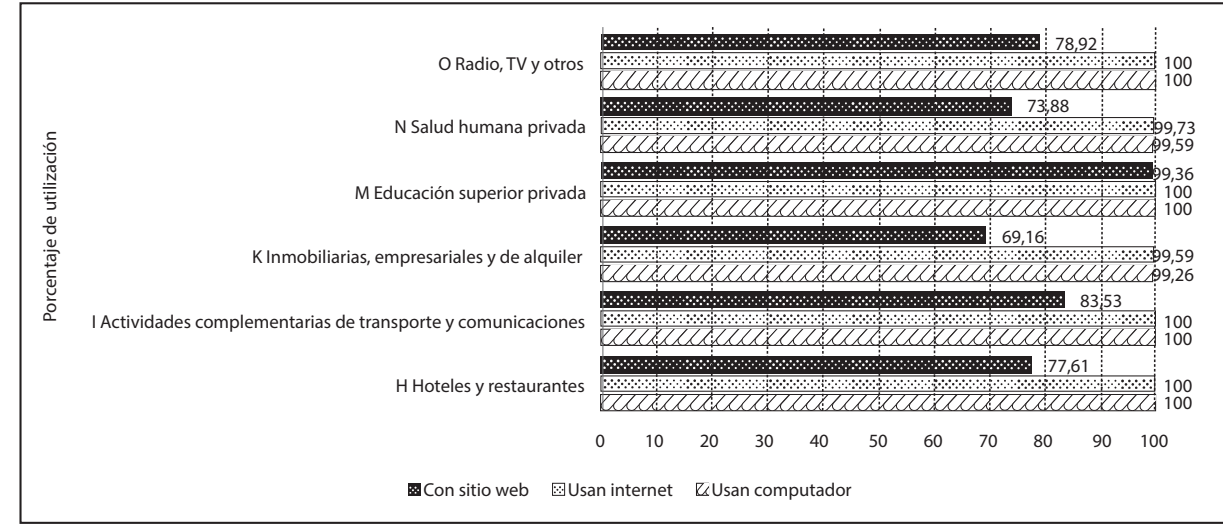

Fuente. DANE, (2012). 
Se presentan los porcentajes de las actividades realizadas por las empresas que usaron internet. En el caso de hoteles y restaurantes, internet se utiliza para la búsqueda de información (94.9\%), banca electrónica $(94.6 \%)$, servicio al cliente $(79.9 \%)$ y transacciones con organismos del Gobierno $(70,1 \%)$, mientras que las transacciones que menos se realizan son distribuir productos en línea $(36.7 \%)$ y contratación interna o externa $(36.6 \%)$.
En el sector, $99 \%$ de las empresas asegura que usa internet para enviar o recibir correos; $96.3 \%$ para búsqueda de información; $95.2 \%$ para banca electrónica, y $82 \%$ para servicio al cliente. Las actividades que menos se realizan son distribuir productos en línea con $32.6 \%$ y contratación interna o externa con $38.1 \%$.

El $76.9 \%$ de las empresas que no usaron internet no lo consideran necesario. No obstante, $53.8 \%$ lo usaría si esto mejorara la gestión administrativa, y $46.2 \%$ también lo haría si ello les facilitara capacitar a su personal (Tabla 1 y figura 2).

Tabla 1. Actividades realizadas por las empresas que usaron internet.

\begin{tabular}{|c|c|c|c|c|c|c|}
\hline $\begin{array}{l}\text { Actividades de uso } \\
\text { de internet. }\end{array}$ & $\begin{array}{l}\text { Hoteles y } \\
\text { restaurantes. }\end{array}$ & $\begin{array}{c}\text { Actividades } \\
\text { complementarias } \\
\text { de transporte y } \\
\text { comunicaciones. }\end{array}$ & $\begin{array}{l}\text { Inmobiliarias, } \\
\text { empresariales y de } \\
\text { alquiler. }\end{array}$ & $\begin{array}{l}\text { Educación } \\
\text { superior } \\
\text { privada. }\end{array}$ & $\begin{array}{l}\text { Salud } \\
\text { humana } \\
\text { privada. }\end{array}$ & $\begin{array}{c}\text { Radio, TV } \\
\text { y otros. }\end{array}$ \\
\hline $\begin{array}{l}\text { Enviar o recibir } \\
\text { correo electrónico. }\end{array}$ & 100 & 100 & 99.9 & 100 & 99.9 & 100 \\
\hline $\begin{array}{l}\text { Búsqueda de } \\
\text { información. }\end{array}$ & 94.9 & 94.3 & 95.1 & 100 & 98.2 & 95.1 \\
\hline Banca electrónica. & 94.6 & 92.8 & 92.6 & 100 & 97.4 & 93.5 \\
\hline $\begin{array}{l}\text { Transacciones con } \\
\text { organismos del } \\
\text { gobierno. }\end{array}$ & 70.1 & 77.4 & 62.2 & 98.7 & 81.7 & 73 \\
\hline Servicio al cliente. & 79.9 & 80.7 & 78.6 & 98.7 & 82.1 & 73.5 \\
\hline $\begin{array}{l}\text { Distribuir } \\
\text { productos en línea. }\end{array}$ & 36.7 & 31.3 & 19.7 & 58.6 & 28 & 21.1 \\
\hline $\begin{array}{l}\text { Recibir pedidos } \\
\text { por internet. }\end{array}$ & 54.8 & 47.8 & 39.1 & 54.8 & 47.5 & 37.8 \\
\hline $\begin{array}{l}\text { Hacer pedidos por } \\
\text { internet. }\end{array}$ & 56.9 & 54.1 & 47.7 & 66.9 & 60.7 & 50.8 \\
\hline $\begin{array}{l}\text { Capacitación de } \\
\text { personal. }\end{array}$ & 45.5 & 60.1 & 49.4 & 75.8 & 48.4 & 44.3 \\
\hline $\begin{array}{l}\text { Contratación } \\
\text { interna o externa. }\end{array}$ & 36.6 & 41.3 & 40.3 & 51 & 32.2 & 27 \\
\hline $\begin{array}{l}\text { Uso de } \\
\text { aplicaciones. }\end{array}$ & 62.5 & 74.7 & 69.2 & 86 & 68.2 & 70.3 \\
\hline $\begin{array}{l}\text { Llamadas } \\
\text { telefónicas por } \\
\text { internet VoIP. }\end{array}$ & 49.4 & 67.6 & 52.7 & 77.1 & 46.5 & 58.4 \\
\hline
\end{tabular}

Fuente. DANE, (2012). 
Figura 2. Intención de uso y causas de empresas que no usaron internet.

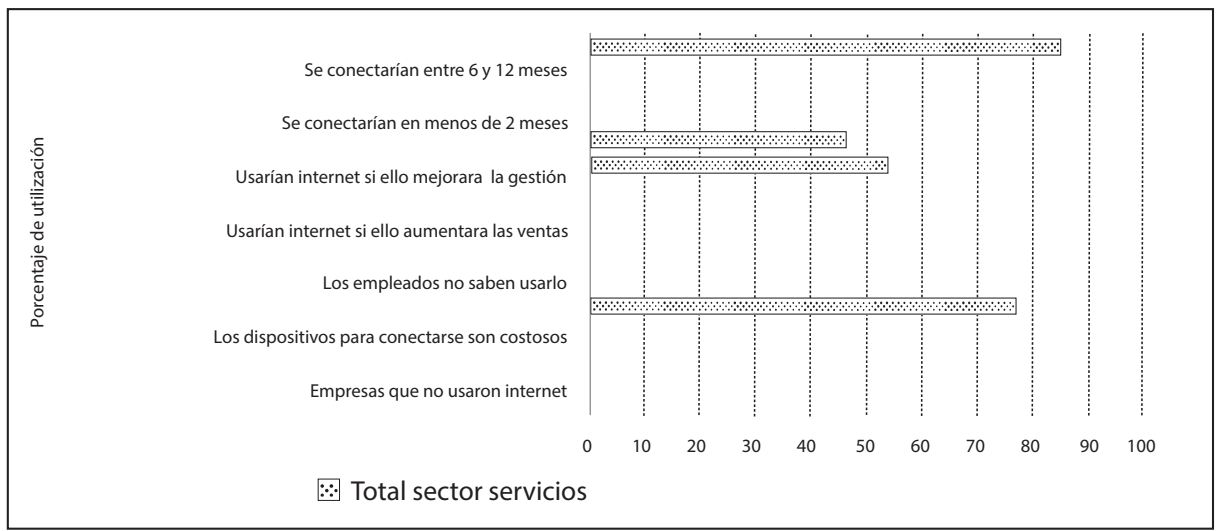

Fuente. DANE, (2012).

Más adelante se muestran los porcentajes de ventas y compras realizadas a través de comercio electrónico. Las actividades de educación superior son las que mayor porcentaje de ventas alcanzan con $15.6 \%$, seguidas de complementarias de transporte y comunicaciones con $13.8 \%$ y de las actividades de hoteles y restaurantes con $11.5 \%$ (Figura 3).
En cuanto a los porcentajes de compras a través de comercio electrónico, complementarias de transporte y comunicaciones con $17.6 \%$ y las de educación superior. En general, los hoteles y restaurantes hacen menor uso de aplicaciones desarrolladas o creadas por la empresa, y descargadas o de uso gratuito.

Figura 3. Ventas y compras a través del comercio electrónico.

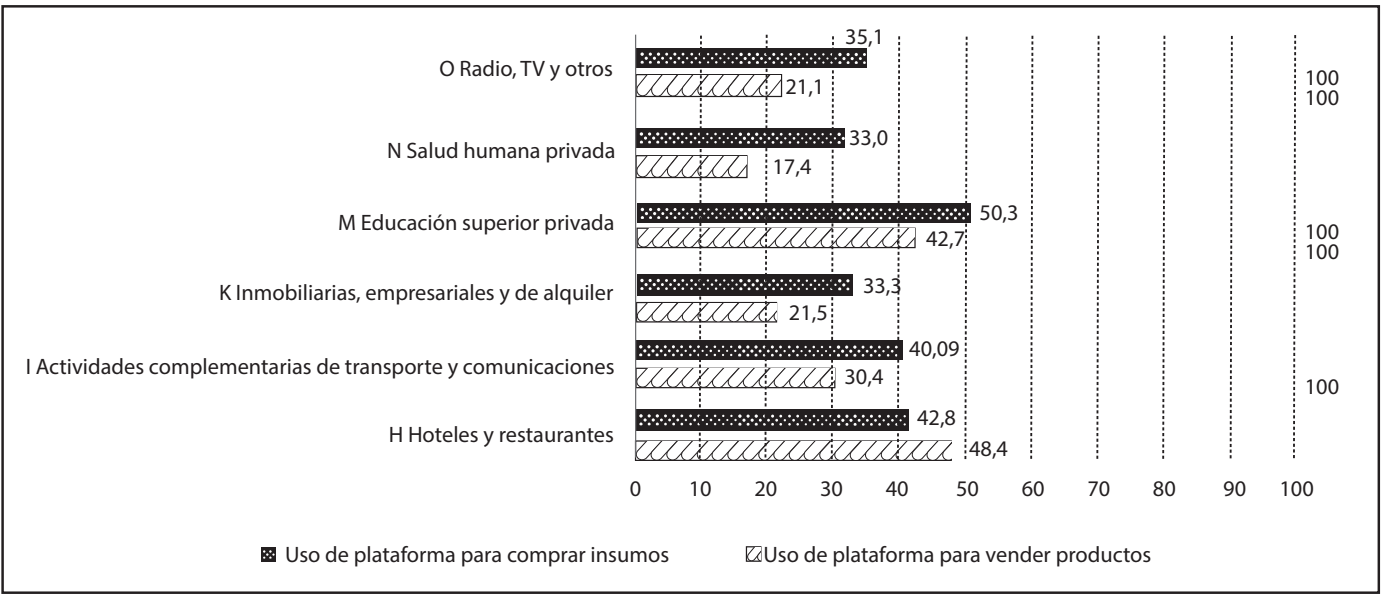

Fuente. DANE, (2012). 


\section{Impacto de las TIC en la competitividad de los hoteles en Bucaramanga}

Se muestran los resultados de la encuesta Saplicada a los hoteles afiliados a Cotelco de Bucaramanga (Tabla 2). La muestra está distribuida entre hoteles desde una estrella hasta cinco estrellas. Más de la mitad de los hoteles se encuentran categorizados entre tres y cuatro estrellas. Los hoteles en Bucaramanga reconocieron la utilidad del uso y la adopción de las TIC. El $90 \%$ de los hoteles encuestados hacen uso de ellas en sus labores diarias y las reconocen como una valiosa herramienta competitiva. Una sinopsis de las principales TIC utilizadas en los hoteles encuestados incluye la dotación de hardware y software de gestión y el uso de tecnologías de conexión a redes y aplicaciones de marketing electrónico y de ventas, entre otros. El software más implementado es gestión de clientes con $76 \%$, seguido de gestión contable con $71 \%$, gestión de personal con $48 \%$ y gestión de almacén con $43 \%$.

Los hoteles utilizan internet para tener una relación más dinámica con el entorno, adoptar estrategias competitivas y brindar un mejor servicio al cliente. Así, el $90 \%$ de los hoteles utiliza internet para comunicación, $81 \%$ para transacciones bancarias, $67 \%$ para e-commerce y búsqueda de información, y $57 \%$ para formación y aprendizaje.
Uno de los canales de venta utilizados es la propia página web del hotel, a través de la cual el establecimiento ofrece y vende sus servicios directamente al cliente. El $95 \%$ de los hoteles afirma tener página web, la cual les permite a los clientes acceder a información, hacer consultas y reservas, entre otras cosas. No obstante, tan solo $11 \%$ de las reservas se realiza a través de estas. Según los hoteles, las principales limitantes para realizar reservas por internet son las formas de pago, la seguridad, la desconfianza de los clientes debido a los problemas logísticos y la incertidumbre legal por la venta. Es decir, la adopción y el uso de las TIC no siempre garantizan el establecimiento de relaciones más estrechas con los clientes.

Más adelante, se presentan los resultados delimpactodeinterneten contactoy captación de clientes, expansión del mercado, ventaja competitiva, imagen y calidad, entre otros, el cual es visto como una medida que genera valor agregado contribuyendo a satisfacer las necesidades de los clientes, aumentando la lealtad, las tasas de ocupación, cautivando nuevos clientes y reduciendo costos, etc. Así, por ejemplo, más del $71 \%$ afirma que internet ha impactado positivamente en la expansión del mercado, la imagen, la calidad, y ha generado una ventaja competitiva. Solo el $38 \%$ de los hoteles considera que les permite reducir costos (Figura 4). 
Tabla 2. Impacto de las TIC en los hoteles afiliados a Cotelco de Bucaramanga.

\begin{tabular}{|c|c|c|c|}
\hline Variable & $\%$ & Variable & $\%$ \\
\hline \multicolumn{2}{|l|}{ Categoría del hotel } & \multicolumn{2}{|l|}{ Reservas de internet } \\
\hline Una estrellas & 19 & Sí & 90 \\
\hline Dos estrellas & 5 & No & 10 \\
\hline Tres estrellas & 33 & \multicolumn{2}{|l|}{ Porcentaje de reserva en sitio propio de internet } \\
\hline Cuatro estrellas & 33 & $0-25$ & 74 \\
\hline Cinco estrellas & 10 & $26-50$ & 11 \\
\hline \multicolumn{2}{|l|}{ Utilización de TIC } & $51-75$ & 5 \\
\hline Sí & 90 & $76-100$ & 11 \\
\hline No & 10 & \multicolumn{2}{|l|}{ Porcentaje de reserva en operadores web. } \\
\hline \multicolumn{2}{|l|}{ Usos de TIC por categoría } & Ninguno & 29 \\
\hline Gestión de clientes & 76 & $1-25$ & 33 \\
\hline Gestión de almacén & 43 & $26-50$ & 29 \\
\hline Gestión de personal & 48 & $51-75$ & $5 \%$ \\
\hline Gestión contable & 71 & $76 \%-100 \%$ & 5 \\
\hline Gestión de restaurante & 38 & \multicolumn{2}{|l|}{ Porcentaje de efectividad de las reservas por internet } \\
\hline \multicolumn{2}{|l|}{ Utilización de internet } & 0 & 10 \\
\hline Comunicación -chat, correo electrónico-. & 90 & $1-25$ & 14 \\
\hline Búsqueda de información & 67 & $26-50$ & 19 \\
\hline Transacciones bancarias & 81 & $50-75$ & 19 \\
\hline e-commerce & 67 & $76-100$ & 38 \\
\hline Formación y aprendizaje & 57 & \multicolumn{2}{|l|}{ Principales limitantes para reservar por internet } \\
\hline \multicolumn{2}{|l|}{ ¿Los hoteles brindan capacitación en TIC? } & Forma de pago & 43 \\
\hline Sí & 62 & Seguridad en los pagos & 33 \\
\hline No & 38 & Incertidumbre del marco legal de la venta & 10 \\
\hline \multicolumn{2}{|l|}{ Tienen página web } & Problemas logísticos -incumplimiento de compromisos-. & 33 \\
\hline Sí & 95 & & \\
\hline No & 5 & & \\
\hline
\end{tabular}

Fuente. Elaboración propia. 
Figura 4. Impacto de internet en los resultados de la empresa.

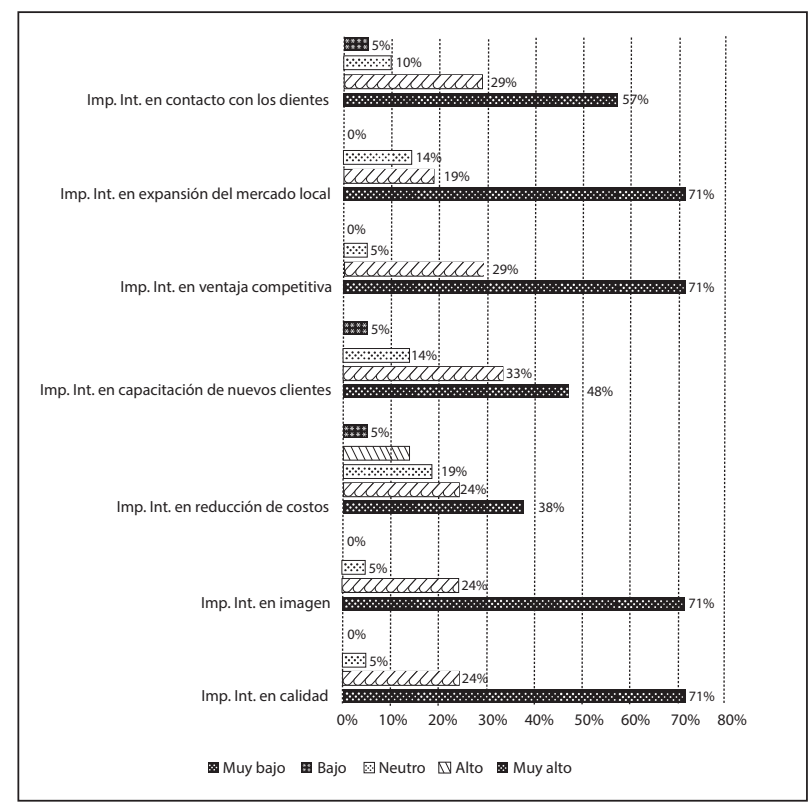

Fuente. Elaboración propia.

\section{Conclusiones}

T os hoteles tienen a su disposición gran que les permiten mejorar la eficiencia de sus procesos internos y la satisfacción de sus clientes. En el sector hotelero de Bucaramanga, el nivel de adopción y uso de las TIC es elevado, principalmente, en hoteles de tres, cuatro y cinco estrellas, que se centra en procesos que generen alto valor agregado.

Las principales TIC utilizadas en los hoteles incluyen la dotación de hardware y software de gestión, tecnologías de conexión a redes, aplicaciones de marketing electrónico y de ventas, lo cual impacta positivamente en la expansión del mercado, la imagen y la calidad; además, genera una ventaja competitiva, sin embargo, no le están sacando todo el potencial a las TIC.
Las TIC pueden ayudar a la empresa a mejorar su eficiencia y a ser más competitiva, no obstante, este proceso debe estar asistido por un esfuerzo de planeación, formación a los empleados en el uso y adopción de las TIC y cambio organizacional.

Entre las principales barreras para la utilización de las TIC por parte de los clientes de los hoteles es la percepción sobre la baja calidad del servicio, la falta de información, los problemas logísticos en el cumplimiento de lo contratado y la inseguridad en pagos vía electrónica. Los hoteles afiliados a Cotelco deberán fortalecer la adopción y el uso de las TIC, así como la confianza de los clientes. 


\section{Referencias}

Alberca Oliver, M. P. y Parte Esteban, L. (2010). Nuevas tecnologías y productividad en las empresas hoteleras: evidencia empírica (2000-2005). Turismo y Desarrollo Local, 3(7).

Bakos, J. Y.y Treacy, M. E. (1986). Information technology and corporate strategy: A research perspective. MIS Quarterly, 10(2), 107-119.

Banco de España. (2010). Summary economic indicators: 1.5 Information And communication technology (ICT): Specific ICT indicators. Spain, EU, and United States. Recuperado de http://www.bde.es/webbde/es/estadis/infoest/ si_1_5e.pdf

Botello Peñaloza, H. A., Pedraza Avella, A. C. y Contreras Pacheco, O. E. (2015). Análisis empresarial de la influencia de las TIC en el desempeño de las empresas de servicios en Colombia. Revista Virtual Universidad Católica del Norte, 2(45), 3-15.

Brown, P. y Stange, K. (2002). Investment in information technology: The multi-billion dollar game of chance. Hospitality Business Review, 4(1), 28-38.

Buhalis, D. y Law, R. (2008). Progress in information technology and tourism management: 20 years on and 10 years after the Internet-The state of eTourism research. Tourism Management, 29(4), 609-623.

Caro Encalada, M. J. (2008). El uso de las tecnologías de la información y comunicación en el sector hotelero de la Península de Yucatán: hacia un modelo explicativo (Tesis doctoral). Universidad Politécnica de Madrid, Madrid, España.

Cipolla, F.; Nicol, E. y Cipolla-Ficarra, M. (2010). Usability, communicability and cultural tourism in interactive systems: trends, economic effects and social impact. En International Workshop on Human-Computer Interaction, Tourism and Cultural Heritage (pp. 100-114). Berlin, Heidelberg: Springer.

DANE (2012). Indicadores básicos de tecnologías de información y comunicación. Bogotá: Departamento Administrativo Nacional de Estadística.

DANE (2012). Indicadores básicos de tenencia y uso de las tecnologías de la información y comunicación en empresas. Recuperado de http://www. dane.gov. co/files/investigaciones/boletines/tic/ prese_tic empresas_2012def.pdf
eBusinessW@tch. (2006). ICT and e-Business in the Tourism Industry. Recuperado de http://ec.europa. eu/enterprise/archives/e-business-watch/studies/ sectors/tourism/documents/Tourism_2006.pdf.

Goldhar, J. y Jelinek, M. (1985). Economías de la variedad basadas en la tecnología. Harvard Deusto Business Review.

González, S. M. y Gidumal, J. B. (2015). Segunda economía en el sector turístico: TIC y puestos de trabajo. Pasos: Revista de Turismo y Patrimonio Cultural, 13(5), 1265-1275.

Hensdill, C. (1998). Hotels technology survey. En Hotels, pp. 51-76.

Holjevac, I. A. (2003). A vision of tourism and the hotel industry in the 21st century. International Journal of Hospitality Management, 22(2), 129-134.

Ibarra Cisneros, M. A., González Torres, L. A. y Cervantes Collado, K. E. (2016). El aprovechamiento de las TIC en empresas pequeñas y medianas de Baja California, México: el caso del sector manufacturero. Revista Internacional de Economía y Gestión de las Organizaciones, $3(1)$.

Jacob, M. y Bravo, A. (2001). Estudio exploratorio sobre innovación en el sector turístico balear. Madrid: Cotec.

Jang, S. y Chen, M. H. (2008). Financial portfolio approach to optimal tourist market mixes. Tourism Management, 29(4), 761-770. doi: 10.1016/j.tourman.2007.09.003

Jo Bitner, M. (2001). Service and technology: Opportunities and paradoxes. Managing Service Quality: An International Journal, 11(6), 375-379.

Kaushik, P.D. y Singh N. (2003). Information Technology and Broad-Based Development: Preliminary Lessons from North India. World Development, 32(4), 591-607. https://doi.org/10.1016/j.worlddev.2003.11.002

Law, R., Leung, D. y Au, N. (2013). Progress and development of information technology in the hospitality industry evidence from Cornell Hospitality Quarterly. Cornell Hospitality Quarterly, 54(1), 10-24.

Lee, S.-C., Barker, S. y Kandampully, J. (2003). Technology, service quality, and customer loyalty in hotels: Australian managerial perspectives. Managing Service Quality: An International Journal, 13(5), 423-432. 
Leyva, B.; Morales, M. y Heredia, J. (2015). Las redes sociales como una alternativa para mejorar la productividad y competitividad de la empresa. Invurnus, 10(1), 3-11.

MacFarlan, F. W. (1985). La tecnología de la información cambia el modo de competir. Harvard Deusto Business Review, 22, 43-50.

Martínez, J., Majó, J. y Casadesús, M. (2006). El uso de las tecnologías de la información en el sector hotelero. En Proceedings of the VI Congress TURITEC: Turismo y tecnologias de la información y las comunicaciones. Málaga: Universidad de Málaga.

Meuter, M. L., Bitner, M. J., Ostrom, A. L., y Brown, S. W (2005). Choosing among alternative service delivery modes: an investigation of customer trial of self-service technologies. Journal of Marketing, 69(2), 61-83. https://doi.org/10.1509/jmkg.69.2.61.60759

Millar, V. y Porter, M. (1986). Cómo obtener ventajas competitivas, por medio de la información. Harvard Deusto Business Review, 25, 3-20.

Mithas, S., Tafti, A. R., Bardhan, I. y Goh, J. M. (2012). Information technology and firm profitability: Mechanisms and empirical evidence. MIS Quarterly, 36(1), 205-224.

Moliner Velázquez, B., Fuentes Blasco, M. y Gil Saura, I. (2015). Las TIC como base de segmentación en el contexto b2b turístico: estudio aplicado en hoteles españoles. Revista de Análisis Turístico, 18, 19-31.

Monroy, C. R. y Ramos, S. R. (2016). Utilización de las TIC y valoración de la competitividad de las empresas turísticas en Guatemala. Transitare, 2(1).

Morcillo Ortega, P. y Bueno Campos, E. (1993). Aspectos estratégicos de la competitividad empresarialun modelo de análisis. En Innovación y competitividad un reto para la empresa de 1993 (vol. 1, pp. 823-848). VitoriaGasteiz: Comunicaciones: VII Congreso AECA.

ONTSI. (2007a). Diagnóstico tecnológico del sector de turismo rural. Observatorio de las Telecomunicaciones y de la Sociedad de la Información. Recuperado de http://www.ontsi. red.es/ontsi/sites/ontsi/files/4_2.pdf

ONTSI. (2007b). Diagnóstico tecnológico del sector hotelero. Observatorio de las Telecomunicaciones y de la Sociedad de la Información. Recupoerado de https://www.ontsi.red.es/ontsi/sites/ontsi/ files/1197382506398.pdf
PwC's (2010). Talent Mobility 2020: The next generation of international assignments. Recuperado de https:// www.pwc.com/gx/en/managing-tomorrows-people/ future-of-work/pdf/talent-mobility-2020.pdf

Reinders, M. J., Dabholkar, P. A., y Frambach, R. T. (2008). Consequences of forcing consumers to use technology-based self-service. Journal of Service Research, 11(2), 107-123. https://doi. org/10.1177/1094670508324297

Rodríguez, J. M., Alonso, M. M., Rubio, L. y Esteban, C. (2008). Conocimiento y aprendizaje en las grandes cadenas hoteleras españolas en Iberoamérica: internet como herramienta de aprendizaje organizativo. Cuadernos de Turismo, 21, 135-157.

Ruiz Molina, M. E., Gil Saura, I. y Moliner Velázquez, B. (2013). Las tecnologías que implantan los hoteles y las tecnologías que gustan a los huéspedes. Revista de Análisis Turístico, 15, 61-70.

Sahadev, S. y Islam, N. (2005). Why hotels adopt ICTs: A study on the ICT adoption propensity of hotels in Thailand. International Journal of Contemporary Hospitality Management, 17(5), 391-401.

Sánchez, C. P., Llano Monelos, P. de y López, M. R. (2016). Las TIC como inductores de competitividad y facilitadores del éxito empresarial. International Journal of Information Systems and Software Engineering for Big Companies (IJISEBC), 3(1), 8-26.

Stamboulis, Y. y Skayannis, P. (2003). Innovation strategies and technology for experience-based tourism. Tourism Management, 24(1), 35-43.

UNWTO (World Tourism Organization) (2014). Tourism Highlights, 2014 edition. UNWTO.

Vilaseca, J. y Torrent, J. (2004). Principis d'economia del coneixement. Barcelona: Editorial UOC.

Wichels, S. (2016). Cómo las redes sociales están cambiando la comunicación hotelera. Revista Internacional de Tecnología, Ciencia y Sociedad, 3(2). 